\title{
Cadena productiva y comercialización de arveja (Pisum sativum L.) del corredor económico en Acobamba, Huancavelica, Perú
}

\author{
Pea production and marketing chain (Pisum sativum L.) \\ of the economic corridor in Acobamba, Huancavelica, Perú
}

Julián Coaquira Incacari ${ }^{1}$, Amelia Huaringa Joaquín ${ }^{2}$, Roberto Coaquira Incacari ${ }^{3 *}$

\section{RESUMEN}

Una cadena productiva es un conjunto de agentes económicos interrelacionados por el mercado desde insumos, producción, transformación y comercialización hasta el consumidor final. En esta cadena se incluye el cultivo de arveja por ser una leguminosa que sirve como alimento ya sea en fresco, grano seco o en productos elaborados como harinas y congelados. Esta experiencia se realizó con la ONG Asociación Civil San Javier del Perú, durante los años 2005-2006, 2006-2007 y 2007-2008 en los distritos de Acobamba, Caja Espíritu, Poma Cocha y Marcas en Huancavelica, Perú. El objetivo fue identificar los agentes que intervienen en la cadena productiva y analizar sus componentes mediante el proyecto "Fortalecimiento de las cadenas productivas" (CAPROHA) financiado por FONDOEMPLEO (MINAGRI). Se utilizó la metodología propuesta por el Ministerio de la Producción que incluye: Motivación, Diagnóstico, Estrategia e Implementación y Evaluación. Como resultado se identificaron los agentes que intervienen en la cadena productiva de arveja verde desde el productor primario hasta el consumidor final. Se observó el interés de los productores en realizar la actividad y por ello decidieron apoyar en la formulación de la propuesta para el desarrollo de las cadenas productivas. Asimismo, se analizaron los componentes de la cadena productiva, se capacitó a los productores sobre los conceptos de competitividad, asociatividad y se enfatizó en la tecnología por la eficiencia y calidad de la producción de arveja. Se concluye que las acciones desarrolladas favorecieron el cambio de actitud de los productores, que descubrieron sus potencialidades, destrezas y lograron valorar sus experiencias, con enfoque en la articulación de actividades económicas, cadenas de valores y productivas.

Palabras clave: cadena productiva, tecnología, asociatividad, competitividad.

\begin{abstract}
A production chain is a set of economic agents interrelated by the market from input, production, transformation, marketing and final consumer. This chain includes the pea crop because it is a legume that serves as food either fresh, dry grain or in processed products such as flour and frozen. These experiences were carried out with the NGO Asociación Civil San Javier del Perú, during the years 2005-2006, 2006-2007, and 2007-2008 in the districts of Acobamba, Caja Espíritu, Poma Cocha and Marcas in Huancavelica, Peru, with the objective of to identify the agents that intervene in the productive chain and analyze the components of the productive chain through the project "Strengthening the Productive Chains" (CAPROHA) financed by FONDOEMPLEO (MINAGRI). The methodology proposed by the Ministry of Production was used, which includes: Motivation, Diagnosis, Strategy and Implementation and Evaluation. As a result, the agents that intervene in the green pea production chain from the primary producer to the final consumer were identified and the interest of the producers in developing the activity was observed and they decided to support the formulation of the proposal for the development of the productive chains. However, the components of the production chain were analyzed, producers were trained on the concepts of competitiveness, associativity and emphasis was placed on technology for the efficiency and quality of pea production. It is concluded that the actions developed favored the change of attitude of the producers, they discovered their potentialities, skills and managed to value their experiences, focusing on the articulation of economic activities, value chains and production.
\end{abstract}

Keywords: productive chain, technology, associativity, competitiveness.

1 Instituto Regional de Desarrollo de la UNALM San Juan de Yanamuclo, Jauja Junín, Perú.

2 Departamento de Fitotecnia, Facultad de Agronomía, Universidad Nacional Agraria La Molina, La Molina, Lima, Perú.

3 Escuela de Posgrado, Universidad Nacional Agraria La Molina, La Molina, Lima, Perú.

* Autor para correspondencia: rcoaquira@lamolina.edu.pe

Fecha de Recepción: 27 de Octubre, 2020.

Fecha de Aceptación: 9 de Febrero, 2021. 


\section{Introducción}

La arveja es una de las legumbres de mayor importancia en el Perú, por su alto valor nutricional, ya que contiene proteínas, vitaminas y minerales como calcio y hierro (FUNIBER, 2017; INIA, 2004; Camarena y Huaringa, 1990; Camarena y Huaringa, 2008). Según el INEI (2013), el cultivo de esta leguminosa, por su extensa distribución en el territorio nacional, ocupa una superficie de 31,214 ha de arveja de grano verde con un rendimiento de $9512 \mathrm{~kg} \mathrm{ha}^{-1}$ (MINAGRI, 2017). Desde el punto de vista social, beneficia económicamente a un gran número de familias por su rentabilidad y su consumo, ya que es una fuente importante de carbohidratos $(6,3 \%$ en grano verde) y proteínas (24,1\% en grano seco) (Milán y Moreira, 1996). Por su buen contenido proteico se convierte, junto con los cereales, en un complemento ideal de la alimentación y, además, mejora la fertilidad de los suelos por su capacidad de fijar el nitrógeno atmosférico (hasta $85 \mathrm{~kg} \mathrm{ha}^{-1}$ por año, según CARITAS, 2004). La arveja tanto para la cosecha en grano seco como en grano verde se localiza mayormente en la sierra, donde se destaca la provincia de Acobamba del departamento de Huancavelica, como una zona productora. El cultivo lo realizan los productores y en la comercialización intervienen otros agentes que compran el producto a un menor precio y luego incrementan el valor. Por ello, una de las alternativas es establecer cadenas productivas para el cultivo de arveja con estos productores.

Por la función que cumple la cadena productiva es un conjunto de actores sociales o grupos de actores sociales involucrados en los diferentes eslabones, como sistema productivo, proveedores de servicio o de insumos, industria de procesamiento, de transformación, agente de distribución y de comercialización, además de consumidores finales del producto y subproducto de la cadena (Gomes, 2003; Llaque, M. y Riveros, H, 2004; Gomes y Valle, 2001). Estos actores desarrollan actividades y se relacionan entre ellos para llevar un producto hasta los consumidores de productos y servicios (Paz, 2002; García, 2005; Kaplinsky, 2000). Algunos actores intervienen directamente en la producción, en la transformación y en la venta del producto, y otros se dedican a brindar servicios. Este conjunto de actores está sometido a la influencia del entorno, representado por varios elementos como las condiciones ambientales o las políticas (Damien, 2006). La cadena productiva tiene como objetivo mejorar la productividad (Gereffi, 2001) desarrollando alianzas estratégicas entre productores organizados, proveedores y comercializadores en el ámbito donde funciona (MINAGRI, 2003). La cadena productiva (Gereffi, 1999) está constituida básicamente por componentes (Villacorta, 2005) y estos son: eslabones, entorno institucional y entorno organizacional. Adicionalmente se plantea analizar la comercialización mediante los corredores económicos (Proyecto PRA, 2001).

- Eslabones: es el conjunto de agrupaciones de actores de la cadena productiva que realizan actividades económicas afines. L eslabones cumplen diversas funciones dentro de la cadena, como producción, transformación, industrialización, comercialización y distribución..

- Entorno institucional: es el conjunto de normas de orden legal, político, económico y social que intervienen en la calidad o en la cantidad de las transacciones que se realizan en una cadena productiva.

- Entorno organizacional: es el conjunto de organizaciones funcionales o territoriales de orden público-privado que tienen capacidad de influir sobre las acciones del ambiente institucional de la cadena productiva.

- Corredores económicos: el concepto de corredor se asocia generalmente a la idea de unir dos puntos distantes entre sí en función de su proyección hacia otros mercados, mediante la mejora del transporte, la energía y las telecomunicaciones. Esto para alcanzar el fortalecimiento de las actividades productivas en todo el trayecto del corredor, cuidando, además, de hacerlo en una forma ambiental y socialmente sostenible.

La comercialización de la arveja se hace mediante canales tradicionales tanto en el mercado mayorista de Huancayo como en el Mercado Mayorista $\mathrm{N}^{\mathrm{o}} 1$ de Lima, que es el mayor centro de abastecimiento de productos agrícolas en Lima Metropolitana. Los productos que se comercializan principalmente son tubérculos, raíces, hortalizas y legumbres. Adicionalmente en la periferia, de manera informal y en menor volumen, se venden abarrotes, arroz, azúcar, 
menestras, etc. Otros centros de expendio son el mercado de productores de Santa Anita y los multimercados como plaza Vea, Metro y Wong (Ministerio de la Producción, 2007).

\section{Materiales y métodos}

Este trabajo se realizó en los distritos de Acobamba, Caja Espíritu, Pomacocha y Marcas, en la provincia de Acobamba, región Huancavelica. El clima en la zona es variado por los diversos pisos altitudinales, desde los 2.100 a $4.200 \mathrm{msnm}$. La zona de producción de arveja verde está comprendida entre los 3.000 y $3.660 \mathrm{msnm}$, que corresponde al piso ecológico quechua-Suni, caracterizado por un clima seco frío cuya temperatura promedio anual es de $12{ }^{\circ} \mathrm{C}$ y mínima de $1{ }^{\circ} \mathrm{C}$. Las precipitaciones pluviales en promedio alcanzan los $600 \mathrm{~mm}$. Por otro lado, la topografía de los terrenos agrícolas es accidentada y en algunos casos agreste con pendientes de 20 a 30\%. El suelo tiene contenido medio de materia orgánica.

\section{Materiales}

Para realizar estas actividades es conveniente tener en cuenta los materiales y recursos que se mencionan a continuación:

- Proyecto ganador Agroideas fue el que financió la movilización de pasantías de los productores locales y nacionales.

- Plan estratégico. Aprobado para formalizar las organizaciones de productores en la provincia de Acobamba (libros de actas y de financiamiento para la legalización de las organizaciones).

- Formato de encuestas. Para el diagnóstico de la situación de actores de arveja.

- Manuales, fichas y materiales de capacitación. Para sensibilización a los productores hacia la organización para el trabajo asociativo.

- Elaboración de cursos y talleres. Para los profesionales, técnicos, especialistas para la capacitación y la asistencia técnica en la formación y en el fortalecimiento de las organizaciones.

- Recursos humanos beneficiados. Productores dispuestos a organizarse en diferentes niveles.

- Logística. Facilita el uso de vehículos, computadoras, videocasete, multimedia, proyector de diapositivas.
- Innovación tecnológica con semillas mejoradas. Difusión y uso de las variedades Alderman, Remate y Early Perfection, uso de tutores y pesticidas.

- Medios de comunicación. Medios divulgativos, afiches, avisos radiales, banderolas, trípticos, etc.

\section{Metodología}

Para formar las cadenas productivas se siguió la metodología propuesta por el Ministerio de la Producción (2007), la cual tiene cuatro fases: Motivación, Diagnóstico, Estrategia e Implementación y Evaluación.

- Motivación. Se aplicó para identificar el potencial de la cadena, ver la demanda y tendencias de mercado, conocer el precio y la rentabilidad del producto, que se alcanzará evaluando la cobertura e impacto de la comercialización. Se realizó convocatoria a empresarios, se identificó a los actores de la cadena.

- Diagnóstico. Análisis sistémico: "Mapping" de la cadena. Se analizaron los puntos críticos, el árbol de problemas y árbol de objetivos.

- Estrategia. Definir objetivos, árbol de problemas y árbol de objetivos, Definir la estrategia de trabajo conjunta, evaluar la negociación y acuerdos (acuerdos, contratos y convenios). Priorizar cuello de botella, identificar posibles alianzas para alcanzar acuerdos y formalizar las organizaciones.

- Evaluación. Fundamental en las decisiones para la implementación por el coordinador general, designación de responsables por eslabón y coordinadores de cadena. Diseño de herramientas de medición de resultados esperados, indicadores de logro, sistema de medición. Responsables.

\section{Resultados y discusión}

\section{Identificación de los actores en la cadena de producción y comercialización}

Se han identificado actores principales o directos y secundarios o indirectos en la cadena de producción y comercialización de arveja en grano verde en la provincia de Acobamba del departamento de Huancavelica. 


\section{Actores directos:}

a) Los productores agropecuarios: las 45 asociaciones que se observan en la Tabla 1 y Figura 1 fueron conformadas por pequeños y medianos productores agrarios que producen $\mathrm{o}$ que intervienen en la producción. Campesinos productores que, como principales agentes de la cadena, coordinan directamente con los otros eslabones y son los proveedores del o los productos de la cadena. En la Tabla 2 se

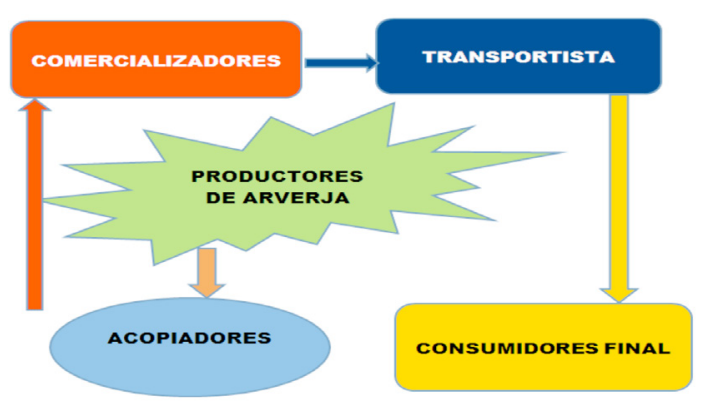

Figura 1. Cadena productiva de arveja. muestran los componentes, actores y funciones de la cadena de producción.

b) Los intermediarios locales o acopiadores: son los agentes de la cadena que intervienen en la compra, acopio y venta de la arveja del productor. Pueden ser minoristas o mayoristas. Estos agentes se caracterizan por la adquisición de la producción para comercializar en el mercado nacional., Entre ellos podemos mencionar al acopiador local, que es el comerciante que opera en la zona de producción y adquiere los productos del pequeño acopiador o directamente de los productores.

c) Los transportistas: son aquellos que acopian de los centros de acopio para transportar hacia el mercado Huancayo y Mercado Mayorista de Lima.

d) Los comercializadores: son aquellos entes, naturales o jurídicos, que compran y venden los productos agrícolas, tanto para el mercado local, regional y nacional, como para exportación, industrial o artesanal. Los mayoristas del Mercado $\mathrm{N}^{\mathrm{o}} 1$ de Lima venden a distribuidores comerciales en ese centro y determinan las

Tabla 1. Comunidades campesinas y asociaciones de agricultores participantes en el proyecto.

\begin{tabular}{lll}
\hline Distrito & Comunidad & \multicolumn{1}{c}{ Asociación } \\
\hline \multirow{4}{*}{ Acobamba } & Allpas & Fortaleza, Dios es Amor, Intihuatana \\
& Acobamba & Huilcapuquio, Huachaccucho y Retoño \\
& Putacca & Los Ángeles de Putacca \\
& Bellavista & Ángeles de Bellavista \\
& Tres de Octubre & Los Andes \\
& Pariahuanca & San José, Inversiones Doloreir \\
& Sachaorcco & Despertad y Fujimori F \\
& Carabamba & Los Tigres y Santa Teresita \\
& Parcostambo & Los Chasquis \\
\hline \multirow{4}{*}{ Pomacocha } & Villarrica & Los Libertadores, Vista Alegre Carhuac \\
& Choclococha & Nueva Esperanza, Willcas, Santiago de Tucuma \\
& Yanacocha & Aguas Negras e Inversiones Yanacocha \\
& Chilcapite & Cuenca San José, Ángeles de Curabamba, Nuevo Amanecer \\
& Incapacchan & Los Incas, Nuevo horizonte \\
& Huilhuic & Reyes Magos \\
& Yanaccara & Santa Cruz \\
& Ayahuasan & Santisima Trinidad, Nuevo Progreso \\
& Pomacocha & Allpa Ruruchic, San Lorenzo \\
\hline Marcas & Pomacancha & Unión Progresista, Nueva Generación, Ventanayuc \\
& Toccto & Santa Rosa \\
\hline \multirow{5}{*}{ Caja Espíritu } & Huanccallaco & Tarpuy, Valle Urubamba \\
& Oyococha & Catamayos, Nuevo Amanecer \\
& Caja Espíritu & San Cristóbal, Virgen de Cucharca \\
\hline
\end{tabular}


Tabla 2. Componentes, actores y funciones de la cadena de producción.

\begin{tabular}{lll}
\hline \multicolumn{1}{c}{ Componentes } & \multicolumn{1}{c}{ Actores } & \multicolumn{1}{c}{ Funciones } \\
\hline Producción agropecuaria & $\begin{array}{l}\text { Productores de arveja de la provincia de } \\
\text { Acobamba. }\end{array}$ & Producir y cosechar. \\
\hline Transporte & Acopiador intermediario (locales y regionales). & Acopiar, almacenar y transportar. \\
\hline Transformadores o agroindustrias & $\begin{array}{l}\text { Industriales (Empresa A\&L Biodiversidad Alto } \\
\text { Andina S.A.C.) }\end{array}$ & Acondicionar, transformar y almacenar. \\
\hline Transportistas & Distribuidor. & Colocar, distribuir y transportar. \\
\hline Comerciantes & Mayoristas (Mercado Mayorista N 1 de Lima) & Almacenar, clasificar, acomodar y vender. \\
\hline Consumo final & y Minoristas. & \\
\hline
\end{tabular}

condiciones de compra de la arveja proveniente de Acobamba, Huancavelica. En la Tabla 3 se muestran los operadores comerciales en el Mercado Mayorista de Lima.

e) Transformadores: son aquellos que transforman o modifican la materia prima. La Empresa A\&L Biodiversidad Alto Andina S.A.C., ubicada en la ciudad de Lima, es la encargada de proporcionar valor agregado o comercializar el producto final a los principales mercados nacionales e internacionales.

f) Consumidores: son individuos u organizaciones con necesidades comunes e interés en determinado producto, para su uso o consumo. Es la fuente primaria de demandas para el mercado de tecnología y el mayor centro de abastecimiento de productos agrícolas en Lima Metropolitana.

\section{Actores indirectos:}

Son aquellos que brindan servicios o insumos en apoyo al desarrollo de la cadena. a) Proveedores de insumos: son normalmente empresas privadas que abastecen de insumos, accesorios, equipos o maquinaria que se requiere para los cultivos, los procesos o la comercialización. Entre ellos tenemos a Hortus S.A., Casa Agropecuaria Montes de Huancavelica.

b) Proveedores de servicios de asistencia técnica: estuvo a cargo del equipo técnico del proyecto Cadenas Productivas de Haba y Arveja (CAPROHA) de la Asociación San Javier del Perú, con la transferencia tecnológica a nivel agronómico, manejo de postcosecha y comercialización a los mencionados beneficiarios ubicados en las provincias de Acobamba, fomentando la capacitación y la extensión permanente.

c) Instituciones financieras: Fondoempleo, Municipalidad Provincial de Acobamba, municipalidades distritales de Pomacocha, Caja Espíritu y Marcas. Ellos proveen de dinero en efectivo necesario para hacer realidad la producción agrícola, su transformación y la

Tabla 3. Operadores comerciales del Mercado Mayorista $N^{\circ} 1$ de Lima.

\begin{tabular}{|c|c|c|}
\hline Nombre del operador & Mercado & Condiciones de compra \\
\hline Juan Ortiz López & \multirow{2}{*}{ Mercado Mayorista $N^{\circ} 1$ de Lima (parada) } & Producto de mediana calidad: Arveja verde \\
\hline Puesto $N^{\circ} 388$ & & Pago al segundo día \\
\hline Carlos Ulloa Urrutia & \multirow{2}{*}{ Mercado Mayorista No 1 de Lima (parada) } & Producto de mediana calidad: Arveja verde \\
\hline Puesto $\mathrm{N}^{\circ} 346$ & & Pago al segundo día \\
\hline Emeterio Ortiz López & \multirow{2}{*}{ Mercado Mayorista $\mathrm{N}^{\circ} 1$ Lima (parada) } & Producto de mediana calidad: Arveja verde \\
\hline Puesto $N^{o} 357$ & & Pago al segundo día \\
\hline
\end{tabular}


comercialización de arveja en grano verde y en grano seco.

\section{Fortalecimiento de las organizaciones de productores}

En el ámbito del proyecto se contaba con agricultores individuales, provenientes de varias unidades con participación en asociaciones de productores debilitados y sin formalización adecuada. Para fortalecer a las comunidades intervenidas y las asociaciones conformadas en el corredor Acobamba-Huancavelica, se realizó la identificación de grupos de productores individuales con deseos de asociarse, para su formalización e inscripción en los registros públicos mediante el apoyo en los siguientes ítems:

- Legalización notarial de la asociación e inscripción en registros públicos.

- Capacitación en aspectos de organización y de gestión empresarial.

- Fortalecimiento de la Federación de Asociaciones de Productores a nivel provincial y apoyo con beneficios concretos a sus bases, facilitando libros de actas y financiamiento respectivo para la legalización.

- Conformación de alianzas estratégicas con diversos actores locales relevantes, con la finalidad de elaborar un plan de acción concertado para solucionar los puntos críticos identificados en la cadena productiva de arveja.

- Pasantías locales y nacionales para conocer experiencias exitosas sobre niveles de organización de productores en la Estación Experimental Donoso Huaral, INIEA Vista Florida, INIEA de Chiclayo, Empresa Procesadora Gandules de Chiclayo, Empresa Santa Natura-Pachacamac, Valle Sagrado de los Incas-Cusco, Empresa Agroindustrias Latino E.I.R.L, INIA AndenesCusco y Mercado Mayorista $\mathrm{N}^{\circ} 1$ de Lima.

- Promoción de la producción de arveja para grano verde.

- Sensibilización a los productores sobre las ventajas y los beneficios de las actividades al formar empresas de tipo asociativo.

- Revalorización de los productos de la zona, enfocándose en los que tienen mayor valor nutritivo para la seguridad alimentaria.

- Aspectos de infraestructura de implementación de nuevos centros de acopio comunales, en cada comunidad del proyecto, y el mejoramiento de infraestructura de cada organización.

\section{Mejoramiento de la producción y de la productividad de la arveja}

En el país tradicionalmente se siembra la arveja con variedades tradicionales y las vainas son pequeñas a medianas y granos de color verde claro. La semilla de tipo americana de vainas y granos de calidad superior permite alcanzar mayor rentabilidad aplicando un manejo agronómico desde la instalación, conducción y cosecha oportuna (Camarena, 2014).

Las principales actividades efectuadas para este mejoramiento fueron:

- Definición del producto mediante la sustitución de semillas locales, muchas de ellas degeneradas y con escaso potencial comercial, por especies traídas de afuera, con mayor demanda y precio en el mercado.

- Capacitación y asistencia técnica en temas como la preparación del terreno, sistemas de siembra en surcos, labores culturales, uso de tutores, control fitosanitario, selección de semillas, uso de variedades comerciales y uso de abonos orgánicos (Bocashi y Biol).

- Instalación de campos de multiplicación de semilla mejorada variedades Alderman, Remate, Early Perfection y Usui.

- Elaboración de un plan de acción concertado con actividades orientadas a la producción con la intervención de instituciones públicas y privada operantes.

- Mercadeo y comercialización. La rueda de negocios en Acobamba entre la FAPAAH y los representantes del Mercado Mayorista $\mathrm{N}^{\circ} 1$ de Lima se llevó a cabo mediante las siguientes acciones.

- Establecimiento de contactos comerciales a través de pasantías a los centros comerciales (Mercado Mayorista $\mathrm{N}^{\mathrm{o}} 1$ de Lima, autoservicios y empresas procesadoras: Santa Natura) con participación de representantes de la FAPAAH y coordinadores distritales.

- Mapeo de las zonas productoras y las fechas probables de cosecha en vaina verde.

- Recolección de información sobre el volumen de la producción familiar por asociación y su consolidación en el ámbito provincial, considerando las variedades existentes. 
- Acompañamiento técnico durante el transporte a Lima y entrega del producto al mercado mayorista.

- Acompañamiento y asistencia técnica en identificación de oportunidades de mercado y organización de la oferta. Mediante contactos y contratos comerciales para organizar la producción en función de la demanda existente en variedades, volúmenes, precios, etcétera.

- Capacitación en cosecha y poscosecha que incluyó formas de recolección en campo e información sobre los parámetros de calidad exigidos por el mercado.

- Financiamiento económico para el viático de los viajes de contacto; un profesional especialista en agronegocios y familias productoras organizadas para la comercialización en volúmenes considerables.

- Registro de volumen de producción de los productos que se ofertarán en el mercado; equipo logístico (vehículos, teléfono, internet, computadoras, multimedia); diseño y elaboración de envases para productos agrícolas; apoyo con camioneta y combustible para el traslado de las cosechas a los centros de acopio.

En la Tabla 4 se observan los campos comerciales de arveja instalados y en la Tabla 5 los campos semilleros conducidos en las campañas agrícolas respectivas.

\section{Estudio de mercados}

La demanda está representada por el Mercado Mayorista $\mathrm{N}^{\circ} 1$ de Lima y es de 26,756 toneladas al año. Esta cantidad supera largamente los actuales niveles de oferta de la provincia de Acobamba (4,500 toneladas/año). La enorme demanda es un incentivo para llevar adelante el negocio. La competencia en cuanto a producción de arveja son las provincias de
Tabla 5. Instalación de campos semilleros expresados en ha.

\begin{tabular}{lccc}
\hline \multirow{2}{*}{ Variedad } & \multicolumn{3}{c}{ Campaña agrícola (ha) } \\
\cline { 2 - 4 } & $2006-2007$ & $2007-2008$ & Total \\
\hline Usui & 3.00 & 2.23 & 5.23 \\
Remate & 1.5 & 1.3 & 2.8 \\
Alderman & 2.0 & 1.55 & 3.55 \\
Total & 6.5 & 5.08 & 11.58 \\
\hline
\end{tabular}

Tayacaja y Churcampa. Huancayo. La competencia en comercialización son los intermediarios de Acobamba, Huancayo y Lima.

\section{Comercialización en grano verde}

La producción de arveja se comercializó en vaina verde para mercado nacional y en grano verde para el exterior.

- Para el reconocimiento de la producción de la arveja de la zona de estudio se realizaron los trámites para la obtención de la "marca" de este producto. Esta denominación se hizo con la participación de los presidentes de las asociaciones de productores de arveja y haba que conforman la Federación de Asociación de Productores Agropecuarios de Acobamba de Huancavelica (FAPAAH).

- La marca tiene por nombre SUMAC RURU y el logotipo: Federación de Asociación de Productores Agropecuarios de Acobamba de Huancavelica (FAPAAH).

- La importancia de la marca se puede determinar desde dos puntos de vista: consumidor y vendedor.

- Para el consumidor es más fácil identificar el producto de su preferencia y así saber exactamente qué recibirá.

Tabla 4. Instalación de los campos comerciales en hectáreas.

\begin{tabular}{lcccr}
\hline \multirow{2}{*}{ Variedad } & \multicolumn{3}{c}{ Campaña agrícola (ha) } & \multirow{2}{*}{ Total } \\
\cline { 2 - 4 } & 2005 a 2006 & 2006 a 2007 & 2007 a 2008 & \\
\hline Usui & 13.81 & 30.25 & 59.44 & 103.50 \\
Remate & 14.81 & 35.55 & 51.29 & 101.65 \\
Alderman & 10.67 & 16.51 & 17.82 & 45.00 \\
Early Perfection & & 5.0 & 4.4 & 9.40 \\
Total & 39.29 & 87.31 & 132.95 & 259.55 \\
\hline
\end{tabular}


- Para el productor-vendedor ayuda a promocionar la arveja sabiendo que el consumidor lo identificará en el punto de venta con mayor facilidad. Asimismo, puede ejercer un mayor control de su participación en el mercado, con respecto a las marcas de la competencia. La utilización de las marcas tiende a aumentar el valor de los productos y da reconocimiento y prestigio directo a la empresa.

- Los parámetros de calidad de la arveja en la comercialización son primera y segunda.

- Primera calidad: maduros fisiológicamente; llenado de granos en la vaina al 90\%; tamaño de vaina de $9 \mathrm{~cm}$, a más; sin daños físicos en el grano y vaina (granizo, helada, sobremanipulación); limpios, sin manchas, ni sobremadurados; libres de enfermedades.

- Segunda calidad: maduros fisiológicamente; llenado de granos en la vaina a menos del $90 \%$; tamaño de vaina menor a $9 \mathrm{~cm}$ de largo; sin daños físicos en el grano y vaina (granizo, helada, sobremanipulación; libres de impurezas (pajas, granilla, tierra). Granos sanos, libres de enfermedades.

- La estrategia de comercialización de arveja en vaina verde estuvo basada en tres acciones básicas: identificar las oportunidades de mercado, identificar y organizar la oferta para esas oportunidades de mercado, y prestar asistencia técnica para obtener un producto en cantidad y calidad.

- En la Tabla 6 se muestran las diferentes etapas durante el proceso de comercialización de la arveja en grano verde, el cual se inicia con la cosecha de las vainas verdes en la chacra o parcelas del productor. Luego el producto es trasladado al centro de acopio comunal, donde se realiza la selección y el embalaje (producto seleccionado, pesado y cerrado). La arveja para ser comercializada es colocada en mallas o yutes a manera de sacos y esto debe realizarse con mucho cuidado, ya que las vainas verdes son muy sensiblesal tratamiento poscosecha. Las variedades de arveja difieren en su resistencia aa la manipulación en estas operaciones, por lo que hay que tener en cuenta este factor al seleccionar una variedad. Además, la arveja es un producto perecible, por lo que la recolección de las vainas en el predio productivo, la selección y su comercialización debe realizarse en el menor tiempo.

Tabla 6. Proceso de comercialización de arveja en grano verde.

\begin{tabular}{ll}
\hline Proceso & Descripción \\
\hline Proceso Productivo & $\begin{array}{l}\text { El proceso productivo consiste en tener la producción de arveja en vaina verde para realizar la } \\
\text { venta para el mercado. }\end{array}$ \\
\hline Acopio & $\begin{array}{l}\text { Esta actividad consiste en llevar la arveja en vaina verde hacia el centro de acopio instalado en } \\
\text { lugares de mayor movimiento de transportes. }\end{array}$ \\
\hline Selección y clasificación & $\begin{array}{l}\text { La selección consiste en separar de las mejores vainas, las vainas enfermas, inmaduras, podridas } \\
\text { y otros. La clasificación consiste en clasificar las vainas de acuerdo al tamaño } 1^{a}, 2^{2} \text {, etc. }\end{array}$ \\
\hline Embalaje & $\begin{array}{l}\text { Después de realizar la selección y la clasificación se hace el ensacado en yutes o mallas lisas (sin } \\
\text { entrenudos) y posteriormente se efectúa el cosido de las mallas o yutes. }\end{array}$ \\
\hline Puesta de marca & $\begin{array}{l}\text { Esto consiste en poner la marca correspondiente en cada malla, con rafias de colores, con el } \\
\text { nombre de la institución y otros. Pero tienen que ser visibles para que no existan confusiones al } \\
\text { momento de descargar en el mercado. }\end{array}$ \\
\hline Pesado & $\begin{array}{l}\text { Cuando las mallas o los yutes estén listos con sus marcas y cosidos respectivos, se pasarán al } \\
\text { pesado de la arveja enmallados. El pesado será en el centro de acopio. }\end{array}$ \\
\hline Transporte & $\begin{array}{l}\text { El transporte y los cargadores se encargarán de recoger las arvejas enmalladas que ya están } \\
\text { pesadas donde el transportista. Serán contratados para el traslado hasta el Mercado Mayorista de } \\
\text { Lima u otros mercados nacionales. }\end{array}$ \\
\hline Mercado Mayorista de Lima & $\begin{array}{l}\text { El transportista descargará el producto en el Mercado Mayorista No 1 de Lima, donde será } \\
\text { distribuido a diferentes mayoristas del mercado de acuerdo a la guía enviada con el transportista. }\end{array}$ \\
\hline Liquidación pago & \begin{tabular}{l} 
La liquidación consiste en el pago a los productores de sus productos enviados hacia el mercado. \\
\hline
\end{tabular} \\
\hline
\end{tabular}




\section{Conclusiones}

Se logró identificar los agentes de la cadena productiva, donde los productores de arveja agrupados en 45 asociaciones en forma organizada llegan a vender su producto a un precio razonable a los mercados de Huancayo y al Mercado Mayorista de Lima, y otra parte a los multimercados como plaza Vea, Metro y Wong.

Se analizó cada componente: en la Producción se logró la adopción e incorporación de variedades, se mejoró la siembra usando tutores, se incidió en la utilización de abono orgánico y uso limitado de pesticidas, desinfección de semillas, control de plagas y enfermedades, control de malezas. En la Transformación se mejoró en la conversión de arveja en grano verde a grano seco. En la Comercialización: las distintas ONG en la provincia de Acobamba desarrollaron proyectos para promover empresas comunales o asociativas que cubrieron la cadena productiva de la arveja.

\section{Recomendaciones}

Se recomienda realizar este tipo de estudios de cadena productiva en otras zonas donde se siembra arveja u otras leguminosas con innovaciones fitotécnicas y fomentar una agricultura hacia un desarrollo sostenible

\section{Literatura Citada}

Camarena. F; Huaringa, A.

1990. El cultivo de arveja. Programa de investigación y Proyección social de Leguminosas de Grano y Oleaginosas. Lima, Perú. 29 p.

Camarena. F; Huaringa, A. 2008. Manual del cultivo de arveja. Lima, Perú. 13 p.

Camarena, F.

2014. Innovación fitotecnia del haba (Vicia faba L.), arveja (Pisum sativum L.) y lenteja (Lens culinaris Medik.). Lima, Perú. 189 p.

CARITAS.

2004. Manual del cultivo de arveja. Caritas Huancayo, INIA, UNCP, Fondo Ítalo Peruano. Huancayo, Perú. 34 p.

Damien van der Heyden.

2006. Guía metodológica para el análisis de cadenas productivas segunda Edición. Quito, Ecuador. 122 p.

INIA.

2004. Cultivo de arveja en los valles del sur chico. Folleto 24. Lima, Perú. 94 p.

FUNIBER.

2017. Base de Datos Internacional de Composición de Alimentos. Fundación Universitaria Iberoamericana. Disponible: https://www.composicionnutricional.com/ alimentos/ARVEJA-TIERNA-5 Consultado: 25/sep/2020.

García, J.

2005. Pymes, Clusters y Cadenas Productivas. Universidad de Lima. Lima, Perú. pp. 10-27.

Gereffi, G.

1999. International Trade and Industrial Upgrading in the Apparel commodity Chain. Journal of International Economics, 48: 37-70

Gereffi, G.

2001. Las cadenas productivas como marco analítico para la globalización. Problemas de Desarrollo, 32(125): 9-37.

Gomes, A. y Valle, S.

2001. Análisis Prospectivo de Cadena Productiva Agropecuaria. Puno, Perú. 34 p.

Gomes de Castro, A, M. Valle, S.M,

2003. Análisis prospectivo de cadena productiva agropecuaria, EMBRAPA. Brasil. 40 p.
INEI.

2013. Resultados definitivos: IV Censo Nacional Agropecuario 2012. MINAGRI. 62 p.

MINAGRI.

2003 Dirección General de Producción Agraria (2003). Cadena productiva de maíz amarillo duro. Valle Chancay Huara, Lima, Perú. 31 p.

Kaplinsky, R.

2000. Spreanding the Gains from Globalization: What can be learned value chain analisis. Documento de trabajo de IDS No. 110. Institute of Development Studies, University of Sussex. Brighton. 37 p.

MINAGRI.

2017. Boletín estadístico de producción agrícola y ganadera, I trimestre 2017. Ministerio de Agricultura y Riego. Lima, Perú. 159 p.

Milan, M.; Moreira, A.

1996. (Pisum sativum L.) En: Meneses. R.; Waaijenberj, H. y Pierola, L. (editores). Las Leguminosas en la Agricultura Boliviana. Revisión de información. Proyecto de Rhizobiología - Bolivia (CIAT, CIF, PNLG - CIFP WAU). Cochabamba, Bolivia. 434 p.

Ministerio de la Producción.

2007. Guía práctica para el desarrollo de cadenas productivas. Lima Perú 198 p.

Llaque, M. y Riveros, $\mathrm{H}$.

2004. Fortalecimiento de la Cadena Maíz- Avicultura en el Valle de Huaura-Perú, Lecciones Aprendidas. Mónica Llaque y Hernando Riveros-. ISSN 0534-5391 No A3/ PE-2004-03. IICA 2004. Lima, Perú. 65 p.

Proyecto de Reducción y Alivio a la Pobreza.

2001. La estrategia de corredores económicos y el proyecto de reducción y alivio a la pobreza. Boletín informativo. (PRA). Lima, Perú. 28 p.

Paz, A.

2002. Plan de Inversiones Cadena de Producción de Semilla de papa Alturas de Mizque. PATER-CASUDE. Bolivia. 43 p.

Villacorta, I.; Quiroga, J.; Zuebieta, J.

2005. Guía para la elaboración de estudios de cadenas productivas locales. PADER- COSUDE. Bolivia. 142 p. 
